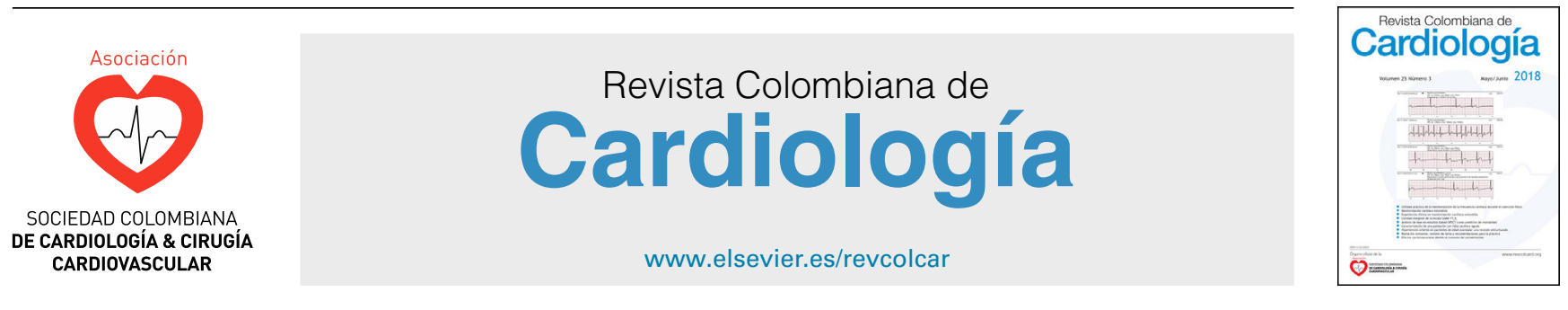

CARDIOLOGÍA DEL ADULTO - REVISIÓN DE TEMAS

\title{
Normoglucemiantes orales y riesgo cardiovascular
}

\section{Guillermo Guzmán a,*, Juan Esteban Gómez ${ }^{\mathrm{b}}$, Leidy Johanna Plaza ${ }^{\mathrm{c}}$ y María Claudia Sánchez ${ }^{c}$}

\author{
a Servicio de Endocrinología, Fundación Valle del Lili, Cali, Colombia \\ b Servicio de Cardiología-Falla Cardiaca, Fundación Valle del Lili, Cali, Colombia \\ c Medicina, Universidad Icesi, Fundación Valle del Lili, Cali, Colombia
}

Recibido el 31 de agosto de 2017; aceptado el 30 de octubre de 2017

Disponible en Internet el 1 de mayo de 2018

\section{PALABRAS CLAVE Riesgo \\ cardiovascular; Antidiabéticos; Empagliflozina; Liraglutida}

\begin{abstract}
Resumen
Introducción: la enfermedad cardiovascular es la principal causa de muerte en el mundo, así como la primera causa de morbilidad y mortalidad en pacientes con diabetes mellitus; por tanto, es importante conocer los diferentes medicamentos que existen hoy para el manejo de la diabetes mellitus y sus efectos, tanto positivos como negativos a nivel cardiovascular. De ahí que las diferentes sociedades y asociaciones científicas del mundo hayan emitido la recomendación de que todos los medicamentos para el tratamiento de la diabetes mellitus tipo 2 deben ser evaluados y certificados como seguros a nivel cardiovascular.

Metodología: se hizo una búsqueda ampliada de la literatura existente acerca de los antidiabéticos actuales y sus efectos cardiovasculares.

Resultados: existen diferentes tipos de medicamentos que se han relacionado con disminución o aumento del riesgo cardiovascular. En la actualidad hay evidencia que relaciona la metformina (biguanida), la empagliflozina (inhibidor del cotransportador sodio- glucosa 2) y la liraglutide (análogo de péptido similar al glucagón) con menos muerte cardiovascular y eventos cardiovasculares en pacientes con enfermedad cardiovascular establecida.

Conclusión: los pacientes con enfermedad cardiovascular conocida pueden tener un beneficio adicional seleccionando medicamentos hipoglucemiantes con un mejor perfil de seguridad cardiovascular.

(c) 2018 Sociedad Colombiana de Cardiología y Cirugía Cardiovascular. Publicado por Elsevier España, S.L.U. Este es un artículo Open Access bajo la licencia CC BY-NC-ND (http:// creativecommons.org/licenses/by-nc-nd/4.0/).
\end{abstract}

Abreviaturas: HTA, Hipertensión arterial; DM, Diabetes Mellitus; HBA1C, Hemoglobina glicosilada; OMS, Organización mundial de salud; IAM, Infarto agudo de miocardio; IM, Infarto de miocardio; ECV, Enfermedad cerebrovascular; MACE, Evento adverso cardiovascular mayor (muerte cardiovascular enfermedad cerebrovascular infarto de miocardio); DPP-4, Dipeptidil Peptidasa-4; GLP-1, Péptido similar al glucagón tipo 1; GIP, Peptido gastrointestinal; SGLT-2, Cotransportador sodio glucosa 2; AMPK, Proteína quinasa activada por Adenosin monofosfato; UKPDS, United Kingdom Prospective Diabetes Study; HBA1C, Hemoglobina glicosilada.

* Autor para correspondencia.

Correo electrónico: guillermoeguzman@gmail.com (G. Guzmán). 


\section{KEYWORDS}

Cardiovascular risk;

Antidiabetics;

Empagliflozin;

Liraglutide

\section{Oral glucose-lowering drugs and cardiovascular risk}

\begin{abstract}
Introduction: Cardiovascular disease is the main cause of death worldwide, as well as the first cause of morbidity and mortality in patients with diabetes mellitus. For these reasons it is important to know the different drugs currently available to manage diabetes mellitus and their positive and negative effects at cardiovascular level. Hence, different scientific societies and associations of the world have issued the recommendation that all drugs for the treatment of type 2 diabetes mellitus must be evaluated and certified as safe at cardiovascular level.

Methodology: An extensive search was carried out on the existing literature on current antidiabetic drugs and their cardiovascular effects.

Results: There are different types of drugs that are associated with a decrease or increase in cardiovascular risk. Currently, there is evidence that associated metformin (biguanide), empagliflozin (sodium-glucose cotransporter 2 inhibitor), and liraglutide (a glucagon-like peptide analogue), with less cardiovascular deaths and cardiovascular events in patients with established cardiovascular disease.

Conclusion: Patients with known cardiovascular disease may have an additional benefit in selecting glucose-lowering drugs with a better cardiovascular safety profile.

(C) 2018 Sociedad Colombiana de Cardiología y Cirugía Cardiovascular. Published by Elsevier España, S.L.U. This is an open access article under the CC BY-NC-ND license (http:// creativecommons.org/licenses/by-nc-nd/4.0/).
\end{abstract}

\section{Introducción}

La enfermedad cardiovascular es la principal causa de muerte en Colombia y en el mundo. En 2010, para la Organización Mundial de la Salud (OMS) representó el 31\% de todas las muertes registradas en el mundo. Las principales enfermedades asociadas a muerte cardiovascular son hipertensión arterial (HTA), enfermedad coronaria y enfermedad cerebrovascular (ECV). Otros factores de riesgo cardiovascular son dislipidemia, obesidad, sedentarismo y tabaquismo, además de los factores genéticos ${ }^{1}$, así como la diabetes mellitus (DM), que se asocia con mayor riesgo de falla cardiaca, enfermedad cardiaca hipertensiva y con aumento de dos veces la mortalidad en hombres y tres en mujeres comparado con no diabéticos, de ahí que se le considera como un equivalente de enfermedad cardiovascular ${ }^{2}$.

En 2015, la Federación Internacional de Diabetes reportó 415 millones de adultos con DM y 317 millones de adultos con intolerancia a la glucosa, lo cual se asoció a 5 millones de muertes ${ }^{3}$, convirtiéndose para la OMS en el tercer factor de riesgo para mortalidad prematura, después de la HTA y el tabaquismo.

La principal causa de mortalidad y morbilidad en pacientes con DM es la enfermedad cardiovascular. Un paciente diabético sin infarto de miocardio (IM) tiene el mismo riesgo de eventos cardiovasculares y mortalidad cardiovascular que un paciente no diabético con $\mathrm{IM}^{4-7}$. De esta manera, sonaría lógico que un control glucémico óptimo redujera complicaciones cardiovasculares y mortalidad cardiovascular; no obstante, lo anterior solo ha demostrado reducir morbilidad sin impacto en la mortalidad ${ }^{8,9}$. De ahí que, el uso de terapias eficaces no solo en control glucémico sino también en reducción de complicaciones cardiovasculares surja como una necesidad para el control de pacientes y recientemente ha partido en dos la historia del tratamiento de la DM. Así, en este artículo se revisa la evidencia y pertinencia de la terapia clásica y vanguardista.

\section{Biguanidas-metformina}

La metformina es el fármaco más usado para el tratamiento de la DM tipo 2. Tiene efecto hipoglucemiante al reducir la producción hepática de glucosa y aumentar la utilización de misma, induciendo la enzima proteína quinasa activada por adenosín monofosfato (AMPK) ${ }^{11}$. Se han documentado beneficios en el control glicémico al reducir la hemoglobina glicosilada (HbA1C) aproximadamente 1,5\% comparado con placebo $^{12}$. El United Kingdom Prospective Diabetes Study (UKPDS) demostró menor riesgo de complicaciones macro y microvasculares en pacientes con sobrepeso que realizaron control glicémico estricto en terapia con metformina ${ }^{8,13}$. El seguimiento a largo plazo (10 años) de esta población confirmó los beneficios de la estrategia intensiva con metformina sobre el control glucémico; principalmente, en dichos pacientes, lográndose una disminución del riesgo relativo de IM (0.67, IC 0,51-0.89, p 0.005), muerte relacionada con DM $(0.70$, IC $0.53-0.92$, p 0.01) y muerte por cualquier causa (0.73. 0.59-0.89, p 0.002). Una revisión de Cochrane en 2005 también concluyó que la metformina fue superior en la disminución de desenlaces asociados con DM, incluyendo mortalidad por todas las causas $(p=0.03)^{14}$. Se puede concluir que la metformina es segura desde el punto de vista cardiovascular al asociarse incluso a disminución de complicaciones de este origen.

\section{Tiazolidinedionas}

Son fármacos sensibilizadores de insulinas, pues su mecanismo de acción se basa en unirse al receptor activado 
por proliferadores de peroxisomas (PPAR) que se expresan principalmente en adipocitos, en el hígado y en menor proporción en el páncreas. La unión de las tiazolidinedionas (TZD) a estos receptores en el páncreas, se traduce en aumento de la síntesis y el contenido de insulina en los islotes pancreáticos, aumento de la expresión de los transportadores de glucosa GLUT-1 y GLUT-4 e incremento de la oxidación de glucosa, logrando así favorecer la utilización de esta última ${ }^{15}$, al reducir la HBA1C en un $0.5-1.4 \%$. Este grupo de fármacos presenta un efecto neutro en cuanto al riesgo de hipoglicemia y producen aumento de peso principalmente por retención de líquidos/edemas, por lo que se han asociado también con incremento de falla cardíaca. La pioglitazona se ha asociado con un supuesto beneficio en cuanto a riesgo cardiovascular, mientras que la rosiglitazona parece estar asociada con mayores eventos adversos cardiovasculares, con aumento significativo en la incidencia de IAM y muerte por enfermedad cardiovascular ${ }^{16-21}$, así como de falla cardiaca y mortalidad por cualquier causa ${ }^{22}$. Por otro lado, el estudio RECORD no encontró diferencias significativas en desenlaces cardiovasculares excepto para incremento en falla cardíaca con el uso de rosiglitazona (HR 2.24, 95\% Cl 1.27-3.97) y el estudio PROactive (pioglitazona), en pacientes de alto riesgo cardiovascular, redujo mortalidad por todas las causas, IM no fatal y ECV (HR 0.84, 0.72-0.98, $p=0.027$ ) (16), por lo que se le ha atribuido un potencial beneficio cardiovascular.

\section{Meglitinidas}

Son secretagogos de insulina que actúan en los canales de potasio dependientes de adenosín trifosfato (ATP) a nivel de las células betapancreáticas. Disminuyen la glicemia postpandrial dependiente de la dosis ${ }^{23}$ y ocasionan menos hipoglucemias postprandiales tardías ${ }^{24}$. Este grupo alcanza una disminución de la HBA1C de $1.5 \%$, aunque es menor con nateglinida ${ }^{23,25}$; su efecto adverso más importante es la hipoglicemia, especialmente en pacientes con función renal comprometida ${ }^{26}$. En cuanto al efecto cardiovascular, se había reportado mayor incidencia de eventos cardiovasculares serios (incluyendo isquemia) con su uso, al compararlo con glibenclamida; sin embargo, cabe aclarar que los pacientes estudiados en el grupo de repaglinida tenían enfermedad coronaria de base más severa que los del grupo de glibenclamida ${ }^{27}$. Al compararlo con metformina, se encontró que la meglitinida resultó menos eficaz en reducir la disfunción endotelial en pacientes no obesos con DM tipo 2 a pesar de tener el mismo control glicémico ${ }^{28}$. En el estudio Left Ventricular Dysfunction in Type 2 Diabetes Mellitus - DYDA-, la repaglinida demostró ser un predictor independiente de disfunción ventricular izquierda después de dos años de seguimiento en paciente sin enfermedad cardiaca de base, aunque hasta ahora no se conozca su rol en la disfunción ventricular izquierda ${ }^{29}$. Pese a que con la evidencia expuesta aún no se puede concluir que las meglitinidas tengan algún efecto cardiovascular negativo que afecte clínicamente a los pacientes con DM tipo 2, este grupo de fármacos sigue siendo una opción de manejo farmacológico como terapia combinada o triple para el tratamiento de la DM tipo $2^{30}$.

\section{Sulfonilureas}

Actúan principalmente al estimular la secreción de insulina por las células betapancreáticas, logrando reducciones de la HbA1c del 1.5 al $2 \%$ siempre que haya un páncreas funcionante ${ }^{31}$. Se demostró que el control estricto con sulfonilureas disminuye el riesgo de complicaciones microvasculares, pero no impacta en la mortalidad relacionada con DM ni en la aparición de IM a 10 años $^{32}$. Su uso comparado con metformina para el tratamiento inicial de diabetes, se ha asociado con aumento de eventos cardiovasculares, mayor incidencia de IM, enfermedad cerebrovascular o muerte, con un número de eventos cardiovasculares de 18,2 y 10,4 por 1.000 personas-año en los usuarios de sulfonilurea y metformina, respectivamente (HR 1,21, 95\% IC $1,13,1,30)^{33}$. Esto se debe a la inhibición de los canales de potasio ATP dependientes miocárdicos o receptores de sulfonilurea $2 \mathrm{~A}$, disminuyendo la respuesta del miocardio a la isquemia ${ }^{32}$, la cual está asociada con arritmias y mayor mortalidad cardiovascular ${ }^{34}$. Por su parte, el estudio Nationwide encontró que la monoterapia con glimeperida, glibenclamida y glipizida parece estar asociada con incremento de la mortalidad cardiovascular en comparación con metformina; sin embargo la gliclazida se asoció con menor riesgo ${ }^{35}$. A lo anterior se suma el riesgo incrementado de hipoglucemia con el empleo de este grupo farmacológico, que limita su uso para el tratamiento de la diabetes. Aunque la evidencia no es contundente, ciertas Sociedades recomiendan evitar el uso de este grupo farmacológico en pacientes de alto riesgo cardiovascular; incluso, guías colombianas recientes no consideran su uso, en especial el de glibenclamida como monoterapia ni como primera elección en terapia de segunda línea ${ }^{36}$.

\section{Inhibidores de la dipeptidil peptidasa 4 (DPP-4)-Gliptinas}

El péptido similar al glucagón 1(GLP1) y péptido gastrointestinal (GIP) son incretinas que estimulan la secreción de insulina con la ingesta. Las enzimas DPP-4 inactivan el GLP-1 y GIP, mientras que entre el $70-80 \%$ del GLP-1 secretado tras la ingesta es degradado antes de alcanzar las células betapancreáticas. Al inhibir esta enzima, las gliptinas aumentan los niveles postprandiales de GLP-1 y GIP, disminuyen la resistencia a la insulina y los triglicéridos postprandiales, estimulan la secreción de insulina, suprimen la secreción de glucagón (inhibe la producción hepática de glucosa), enlentecen el vaciamiento gástrico, reducen el apetito y mejoran la función y el número de células betapancreáticas a largo plazo $^{31}$. El Trial Evaluation Cardiovascular Outcomes with Sitagliptin (TECOS) que incluyó pacientes con DM2 y enfermedad cardiovascular instaurada, no demostró diferencia en la incidencia de eventos cardiovasculares como muerte cardiovascular, IAM no fatal, enfermedad cerebrovascular (ECV) y hospitalización por angina inestable o por de falla cardiaca $^{37}$. El Saxagliptin Assessment of Vascular Outcomes in patients with diabetes trial (SAVOR-TIMI 53) incluyó pacientes de alto riesgo y demostró que la saxagliptina no fue inferior a placebo en la incidencia de eventos cardiovasculares; sin embargo, hubo mayor cantidad de eventos de falla cardiaca (HR 1.27 e IC 95\% de 1.07- 1.51 ; p 0.007) ${ }^{38}$. En 
Tabla 1 Grupos farmacológicos para el tratamiento de la diabetes mellitus

\begin{tabular}{llll}
\hline Grupo farmacológico & & Medicamentos \\
\hline Biguanidas & Metformina & & Glimepirida \\
Sulfonilureas & Glibenclamida & Nateglinida & Glipizida \\
Meglitinidas & Repaglinida & Rosiglitazona \\
Tiazolidinedionas & Pioglitazona & & Sitagliptina \\
Inhibidores de la $\alpha$-glucosidasa & Acarbosa & Vildagliptina \\
Inhibidores de la & Miglitol & Alogliptina \\
DPP-4 & Saxagliptina & Einagliptina & Empagliflozina \\
Agonistas dopamina-2 & Bromocriptina & Albiglutide \\
Inhibidores del cotransportador SGLT2 & Canagliflozina & Liraglutide & Dulaglutide \\
Agonistas del & Exenatide & & \\
receptor GLP-1 & Lixieatide & &
\end{tabular}

un estudio de no inferioridad con alogliptina no se evidenció aumento de muerte por causas cardiovasculares, incidencia de IM y ECV comparado con placebo en pacientes diabéticos con antecedente de IM o angina inestable en los 15 a 90 días previos al inicio del fármaco. En general, los iDPP4 son medicamentos de baja potencia para el control glucémico, con un efecto neutro en la perspectiva cardiovascular.

\section{Agonistas GLP-1}

Pertenecen al grupo de las incretinas, hormonas endógenas secretadas por las células $L$ del intestino delgado tras la ingesta de alimentos, y que actúan sobre las células beta del páncreas estimulando la secreción de la insulina. Los agonistas del GLP-1 se unen a los receptores GLP-1 de las células beta del páncreas estimulando la secreción de insulina ${ }^{31}$. Este grupo farmacológico ha tomado gran importancia en el tratamiento de la DM al demostrar su beneficio frente a eventos cardiovasculares ${ }^{39,40}$. El estudio LEADER (liraglutide), en el que $81 \%$ de la población tenía enfermedad cardiovascular conocida, demostró una reducción significativa en desenlaces cardiovasculares mayores (MACE) (HR 0.87; 95\% Cl, 0.78-0.97; $\mathrm{p}<0.001, \mathrm{p}=0.01$ para superioridad), muerte cardiovascular ( $\mathrm{HR} 0.78 ; 95 \% \mathrm{Cl}, 0.66-0.93$; $\mathrm{p}=0.007$ ) y muerte por cualquier causa (HR $0.85 ; 95 \% \mathrm{Cl}$, $0.74-0.97 ; p<0.001)$, sin diferencia significativa para IM no fatal, ECV y falla cardiaca ${ }^{41}$. Entre tanto, el semaglutide demostró reducción del MACE (HR 0.74; 95\% IC, 0.58-0.95; $\mathrm{p}<0.001$ para no inferioridad; $\mathrm{p}=0.02$ para superioridad) y ECV no fatal (HR 0.61; 95\% IC, 0.38-0.99; $\mathrm{p}=0.004$ ), sin reducción significativa para muerte cardiovascular, IM no fatal y falla cardiaca, con incremento significativo en retinopatía (HR 1.76; 95\% IC, 1.11-2.78; $\mathrm{p}=0.02)^{42}$. Otros medicamentos como lisixenatide fallaron en demostrar disminución del MACE (HR 1.02; 95\%, 0.89 a 1.17) ${ }^{40}$, mientras que el exenatide (Exenatide Study of Cardiovascular Event Lowering Trial - EXSCEL) no demostró reducción de mortalidad cardiovascular ${ }^{43}$. Lo anterior sugiere que la reducción de eventos cardiovasculares (MACE) no es de clase terapéutica y que el liraglutide se postula como el medicamento con el mejor perfil de seguridad de este grupo.

\section{Inhibidores del cotransportador sodio glucosa 2 (SGLT2)}

Este grupo de fármacos tiene efecto glucosúrico, que se traduce en disminución de la HBA1C de un $0.5-0.7 \%{ }^{26}$. Su perfil cardiometabólico con disminución de triglicéridos, aumento de HDL, mejoría en la resistencia en la insulina y por ende aterosclerosis y perfil hemodinámico más disminución de la tensión arterial, le confieren a este grupo una expectativa alta en la reducción de eventos cardiovasculares ${ }^{44}$, hecho que se confirmó a través del estudio de empagliflozina (EMPA-REG OUTCOME) en pacientes con DM2 y enfermedad cardiovascular existente, donde se observó una disminución en IM, ACV y muerte cardiovascular del $14 \%$ y de muerte cardiovascular por si sola del $38 \%^{10,44}$. La canagliflozina en el estudio CANVAS redujo la incidencia del conjunto de descenlace constituido por muerte cardiovascular, IM no fatal y ECV no fatal (HR 1.27; IC 95\% 0.75-0.97; $\mathrm{p}<0.001$ para no inferioridad, $\mathrm{p}=0.02$ para superioridad), sin alcanzar reducción individual de muerte cardiovascular, ECV e IAM. De otro lado, se observó aumento de amputaciones (6.3 vs. $3.4 \%$, $\mathrm{p}<0.001)$ y fracturas $(15.4 \text { vs. } 11.9 \% \mathrm{p}=0.02)^{45}$.

Además del perfil cardiometabólico y hemodinámico favorable de estos medicamentos, hay otras teorías que se asocian a sus efectos cardiovasculares favorables, como lo es la teoría de la cetogénesis leve, ya que los inhibidores de SGLT2 derivan el metabolismo de glucosa a oxidación de ácidos grasos, generando aumento de cetonas. El corazón ávidamente extrae cuerpos cetónicos para producir ATP, por lo que la oxidación de estos aumenta la eficiencia muscular cardíaca y esto se ve reflejado en reducción de mortalidad y morbilidad cardiovascular ${ }^{44}$. Este parece ser un probable efecto de clase terapéutica; no obstante, cada medicamento muestra un perfil de seguridad diferente en los estudios clínicos y hacen falta estudios adicionales de seguridad cardiovascular que incluyan el resto de moléculas.

Al ser la enfermedad cardiovascular la principal causa de muerte en personas con diabetes, obliga a un conocimiento estricto del perfil de seguridad cardiovascular de cada uno de los medicamentos disponibles para el control de la diabetes, para determinar la elegibilidad de estos. De ahí que la metformina continúe siendo el medicamento de elección en diabetes, por su buen efecto antihiperglicémico, sin riesgo de hipoglicemia, y porque ayuda a disminuir el riesgo 
Tabla 2 Estudios clínicos de diabetes mellitus y seguridad cardiovascular

\begin{tabular}{|c|c|c|c|c|c|c|c|c|c|c|c|}
\hline \multirow[t]{2}{*}{ Medicamento } & \multicolumn{2}{|l|}{$\begin{array}{l}\text { Inhibidores de } \\
\text { SGLT-2 }\end{array}$} & \multicolumn{3}{|l|}{$\begin{array}{l}\text { Agonistas de } \\
\text { GLP1 }\end{array}$} & \multicolumn{4}{|c|}{$\begin{array}{l}\text { Inhibidores de } \\
\text { DPP-4 }\end{array}$} & \multicolumn{2}{|c|}{ Tiazolidinedionas } \\
\hline & Empagliflozina & Canagliflozina & Lixisenatide & Liraglutide & Semaglutide & $\overline{\text { Exenatide }}$ & Saxagliptina & Alogliptina & Sitagliptina & Pioglitazona & Rosiglitazona \\
\hline Estudio & EMPA-REG & CANVAS & ELIXA & LEADER & SUSTAIN & EXSCEL & SAVOR & EXAMINE & TECOS & PROACTIVE & RECORD \\
\hline \# pacientes & 7.020 & 10.142 & 6.068 & 9.340 & 3.297 & 14.752 & 16.492 & 5.400 & 14.671 & 5.238 & 4.447 \\
\hline $\begin{array}{l}\text { Seguimiento } \\
\text { (años) }\end{array}$ & 3.1 & 3.6 & 2.1 & 3.8 & 2.1 & 3.2 & 2.1 & 1.5 & 2.8 & 4 & 5.5 \\
\hline Edad (años) & 63 & 63.3 & 60 & 64.3 & 64.6 & $65-75$ & 65 & 61 & 66 & 61.8 & $57-59$ \\
\hline $\begin{array}{l}\text { DM (evolución } \\
\text { en años) }\end{array}$ & $>10$ & 13.5 & 9.3 & 12.8 & 13.9 & 12 & 10 & 7.2 & 9.4 & $9.5+/-7$ & $6-7.9$ \\
\hline HbA1C (\%) & 8.1 & 8.2 & 7.7 & 8.7 & 8.7 & 8.0 & 8.0 & 8.0 & 7.3 & 8.1 & 7.8 \\
\hline ECV previa (\%) & 99 & 65 & 100 & 81 & 83 & 13 & 78 & 100 & 100 & 100 & $15 \%$ \\
\hline $\begin{array}{l}\text { Tipo de } \\
\text { enfermedad } \\
\text { cardiovascular }\end{array}$ & $\begin{array}{l}\text { IAM, IC, ECV, } \\
\text { EAP }\end{array}$ & $\begin{array}{l}\text { IAM, IC, ECV, } \\
\text { EAP }\end{array}$ & SCA $<180$ días & $\begin{array}{l}\geq 50 \text { años }+ \\
60 \text { años }+\geq\end{array}$ & $\operatorname{ERC} 0 \geq$ & $\begin{array}{l}\text { ECV } \\
\text { IAM } \\
\text { SCA } \\
\text { EAP }\end{array}$ & $\begin{array}{l}\geq 40 \text { años }+ \\
E C V \text { o } \geq 55 \\
\text { años }+\geq 1 F R\end{array}$ & SCA $<90$ días & IC, ECV, EAP & IAM, RVM, SCA & $\begin{array}{l}\text { Muerte CV, } \\
\text { hospitaliza- } \\
\text { ción CV, IAM, } \\
\text { ECV, IC }\end{array}$ \\
\hline MACE & $\begin{array}{l}0.86 \\
0.74,1.09\end{array}$ & $\begin{array}{l}0.86^{*} \\
0.75,0.97\end{array}$ & $\begin{array}{l}1.02 \\
0.89,1.17\end{array}$ & $\begin{array}{l}0.87^{\circ} \\
0.78,0.97\end{array}$ & $\begin{array}{l}0.74 \\
0.58,0.95\end{array}$ & $\begin{array}{l}0.91 \\
0.83 \\
1.00\end{array}$ & $\begin{array}{l}1.0 \\
0.89,1.08\end{array}$ & $\begin{array}{l}0.96 \\
\leq 1.16\end{array}$ & $\begin{array}{l}0.98 \\
0.89,1.08\end{array}$ & NR & NR \\
\hline Muerte CV & $\begin{array}{l}0.62 \\
0.49,0.77\end{array}$ & $\begin{array}{l}0.87 \\
0.72,1.06\end{array}$ & $\begin{array}{l}0.98 \\
0.78,1.22\end{array}$ & $\begin{array}{l}0.78^{*} \\
0.66,0.93\end{array}$ & $\begin{array}{l}0.98 \\
0.65,1.48\end{array}$ & $\begin{array}{l}0.88 \\
0.76 \\
1.02\end{array}$ & $\begin{array}{l}1.03 \\
0.87,1.12\end{array}$ & $\begin{array}{l}0.79 \\
0.60,1.04\end{array}$ & $\begin{array}{l}1.03 \\
0.89,1.19\end{array}$ & NR & $\begin{array}{l}0.84 \\
0.59,1.08\end{array}$ \\
\hline IAM no fatal & 0.67 & 0.85 & 1.03 & 0.88 & 0.74 & 0.97 & 1.95 & 1.08 & 0.95 & 0.83 & 1.14 \\
\hline
\end{tabular}

n: Numero.
HDAC: Hemoglobina glicosilada

Enf. CV: Enfermedad cardiovascular

IAM: Infarto agudo de miocardio

IC: Insuficiencia card

ECV: Enfermedad cerebrovascular

PVD: Enfermedad vascular periférica

ERC: Enfermedad renal crónica

SCA: Síndrome coronario agud

RAC: Revascularización de arteria coronaria

HTA: Hipertensión arterial

ivascular Outcomes, and Mortality in Type 2 Diabetes

Dment Stud

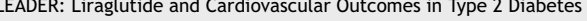

SUSTAN: Semaglutide and Cardiovascular Outcomes in Patients with Type 2 Diabetes

SAVOR: Saxagliptin and Cardiovascular Outcomes in Patients with Type 2 Diabetes Mellitus

EXAMINE: Alogliptin after acute coronary syndrome in patients with type 2 diabetes.

TECOS: Effect of Sitagliptin on Cardiovascular Outcomes in Type 2 Diabetes

oral agent combination therapy for type 2 diabetes

Mace: Evento cardiovascular mayor (muerte cardiovascular, infarto no fatal y ECV no fatal)

IAM: Infarto agudo de miocardio

IC: Insuficiencia cardiaca

V: Cardiovascular

ERC: Enfermedad renal crónica
SCA: Síndrome coronario agudo

NR: No reportado

Estadisticamente significativo

Cualquier reporte de falla cardiaca 
micro y macrovascular. De igual forma, existen otros grupos de medicamentos que se convierten en una buena alternativa para el tratamiento de estos pacientes; entre ellos se mencionan, agonistas de GLP1, en especial liraglutide, el cual demostró una reducción significativa en desenlaces cardiovasculares mayores, muerte cardiovascular y muerte por cualquier causa; sin embargo, no se ha comprobado el mismo beneficio con el resto de medicamentos del mismo grupo, de modo que no se puede afirmar por el momento, que el beneficio corresponda a un hecho de clase. Adicionalmente, la empagliflozina ha demostrado disminución de IAM, ECV y muerte cardiovascular en pacientes con diabetes y a pesar de que hacen falta estudios, esto parece corresponder a un efecto de clase.

Por otro lado, a pesar de su buen efecto antihiperglicémico, las guías recientes, fundamentadas en la evidencia, no recomiendan el uso de sulfonilureas como monoterapia ni como primera opción en terapia combinada, dado el riesgo de hipoglicemia y el impacto en enfermedad cardiovascular, pues su uso se ha asociado con mayor mortalidad cardiovascular al compararlas con metfomina.

Aunque no hay evidencia contundente en contra, es controversial el impacto de las tiazolidinedionas, en especial rosiglitazona, ya que referencias han demostrado su asociación con deterioro de falla cardiaca, aumento significativo en la incidencia de IAM y muerte por enfermedad cardiovascular, lo cual limita su uso para el tratamiento de pacientes con diabetes. Así mismo, se ha encontrado mayor incidencia de eventos cardiovasculares, al comparar meglitinidas con metformina y aparentemente con glibenclamida; pero, aun hace falta literatura concluyente acerca de la seguridad cardiovascular de este grupo de fármacos.

\section{Conclusión}

En la actualidad existen múltiples medicamentos antidiabéticos orales (tabla 1), por lo que al momento de escoger la farmacoterapia se debe individualizar cada paciente, teniendo en cuenta características específicas como peso corporal, función renal, edad y riesgo cardiovascular, a sabiendas de que este último representa gran parte de la mortalidad en personas diabéticas.

Hoy se conoce que la liraglutida (agonista GLP-1) y la empagliflozina (inhibidor del SGLT2) se asocian con una disminución de IM, IM no fatal y muerte cardiovascular (tabla 2), por lo que los pacientes de alto riesgo cardiovascular idealmente deberían recibir alguno de estos medicamentos en combinación con metformina, siempre que no haya contraindicación para ninguno de ellos, teniendo en cuenta que esta última sigue siendo el pilar fundamental en el manejo de la DM2, no solo por su eficacia sino por su perfil de seguridad cardiovascular y su bajo costo.

\section{Conflictos de interés}

Ninguno.

\section{Bibliografía}

1. Mahmooda SS, Levy D, Vasan RS, Wang TJ. The Framingham Heart Study and the epidemiology of cardiovascular diseases:
A historical perspective. Lancet [Internet]. 2014;383:1933-45. Disponible en: http://www.ncbi.nlm.nih.gov/pmc/ articles/PMC4159698/pdf/nihms588573.pdf.

2. Kannel WB, McGee DL. Diabetes and glucose tolerance as risk factors for cardiovascular disease: the Framingham study. Diabetes Care. 1979;2:120-6.

3. International Diabetes Federation. ATLAS de la DIABETES de la FID [Internet]. International Diabetes Federation. 2013. 14 p. Disponible en: http://www.fmdiabetes.org/ $\mathrm{fmd} / \mathrm{des}$ /SP_6E_Atlas_Full.pdf.

4. Serra Sansone MP. Antidiabéticos orales y riesgo cardiovascular. Rev Urug Cardiol. 2012;27:162-74.

5. Marty IJ. Third Report of the National Cholesterol Education Program (NCEP) Expert Panel on Detection. Evaluation, and Treatment of High Blood Cholesterol in Adults (Adult Treatment Panel III) Final Report. Development. 2010.

6. De Backer G, Ambrosioni E, Borch-Johnsen K, Brotons C, Cifkova R, Dallongeville J, et al. European guidelines on cardiovascular disease prevention in clinical practice: third joint task force of European and other societies on cardiovascular disease prevention in clinical practice (constituted by representatives of eight societies and by invit. Eur J Cardiovasc Prev Rehabil [Internet]. 2003;10:S1-10. Disponible en: http://www.ncbi.nlm.nih.gov/pubmed/14555889.

7. From M, Heart C, With D, Subjects D, Prior W, Infarction M. Mortality from coronary heart disease in subjects with type 2 diabetes and in nondiabetic subjects with and without prior myocardial infarction. 1998:229-34.

8. King P, Peacock I, Donnelly R. The UK prospective diabetes study (UKPDS): clinical and therapeutic implications for type 2 diabetes. $\mathrm{Br} \mathrm{J}$ Clin Pharmacol [Internet]. 1999;48:643-8. Disponible en: https://www.ncbi.nlm.nih.gov/ pmc/articles/PMC2014359/.

9. Of S, Carediabetes M. standards of medical care in diabetes-2017 - Diabetes Pro. 2017:40.

10. Brown CE, Badie B, Barish ME, Weng L, Julie R, Chang W, et al. American Association of Clinical Endocrinologists and American College of Endocrinology: clinical practice guidelines for developing a diabetes mellitus comprehensive care plan-2015. 2016;21:4062-72.

11. Zhou G, Myers R, Li Y, Chen Y, Shen X, Fenyk-melody J, et al. Role of AMP-Activated Protein Kinase in Mechanism of Metformin Action Role of AMP-activated protein kinase in mechanism of metformin action. J Clin Invest. 2001;108:1167-74.

12. Holman R, Sanjoy P, Bethel A, Matthews D, Neil A. 10-year follow-up of intensive glucose control in type 2 diabetes. N Engl J Med. 2008;359:1577-89.

13. Lamanna C, Monami M, Marchionni N, Mannucci E. Effect of metformin on cardiovascular events and mortality: a metaanalysis of randomized clinical trials. Diabetes Obes Metab. 2011;13:221-8.

14. Sáenz A, Fernández-Esteban I, Mataix A, Ausejo Segura M, Roqué i, Figuls $M$, et al. Metformin monotherapy for type 2 diabetes mellitus. Cochrane Database Syst Rev. 2005:CD002966.

15. Carlos A. Tiazolidinedionas. Beneficios y riesgos reales. Rev Endocrinol y Nutr [Internet]. 2002;10:69-76. Disponible en: http: / /scielo.sld.cu/scielo.php?script=sci_arttext\&pid=S086421251997000600014

16. Dormandy JA, Charbonnel B, Eckland DJ, Erdmann E, MassiBenedetti M, Moules IK, et al. Secondary prevention of macrovascular events in patients with type 2 diabetes in the PROactive Study (PROspective pioglitAzone Clinical Trial In macroVascular Events): a randomised controlled trial. Lancet [Internet]. 2005;366:1279-89. Disponible en: http://www. sciencedirect.com/science/article/pii/S0140673605675289.

17. Defronzo RA. From the triumvirate to the ominous octet: A new paradigm for the treatment of type 2 diabetes mellitus. Diabetes. 2009;58:773-95. 
18. Wolski K, Nicholls SJ, Nissen SE. Pioglitazone and risk of cardiovascular events in patients with type 2 diabetes mellitus. 2015;298(10.).

19. Home PD, Pocock SJ, Beck-Nielsen H, Curtis PS, Gomis R, Hanefeld $M$, et al. Rosiglitazone evaluated for cardiovascular outcomes in oral agent combination therapy for type 2 diabetes (RECORD): a multicentre, randomised, open-label trial. Lancet [Internet]. 2009;373:2125-35. Disponible en: https://doi.org/10.1016/S0140-6736(09)60953-3.

20. Bae JC. Diabetes Drugs and Cardiovascular Safety. Endocrinol Metab. 2016;31:239-44.

21. Christopher D, Kieny PD, et al. Effect of rosiglitazone on the risk of myocardial infarction and death from cardiovascular causes. N Eng J Med. 2014;356:1-3. Diponible en: http://www.nejm.org/doi/pdf/10.1056/NEJMoa072761.

22. Winkelmayer WC, Setoguchi S, Levin R, Solomon DH. Comparison of cardiovascular outcomes in elderly patients with diabetes who initiated rosiglitazone vs. pioglitazone therapy. Arch Intern Med. 2008;168:2368-75.

23. Blickl JF. Meglitinide analogues: a review of clinical data focused on recent trials. Diabetes Metab. 2006:113-20.

24. Fisman EZ, Tenenbaum A. A cardiologic approach to non-insulin antidiabetic pharmacotherapy in patients with heart disease. Cardiovasc Diabetol. 2009;8:38.

25. Nathan DM, Buse JB, Davidson MB, Ferrannini E, Holman RR, Sherwin R, et al. Medical management of hyperglycemia in type 2 diabetes: A consensus algorithm for the initiation and adjustment of therapy. Diabetes Care. 2009;32:193-203.

26. Garber AJ, Abrahamson MJ, Barzilay JI, Blonde L, Bloomgarden ZT, Bush MA. AACE/ACE Consensus Statement consensus statement by the American Association of Clinical Endocrinologists and American College of Endocrinology on the comprehensive type 2 diabetes management algorithm - 2016 executive summary. 84 Endocr Pract. 2016;22:84-113.

27. Fisman EZ, Tenenbaum A. Non-insulin antidiabetic therapy in cardiac patients: current problems. 2008;45:154-70.

28. Lund SS, Tarnow L, Stehouwer CDA, Schalkwijk CG, Teerlink T, Gram J, et al. Impact of metformin versus repaglinide on nonglycaemic cardiovascular risk markers related to inflammation and endothelial dysfunction in non-obese patients with type 2 diabetes. Eur J Endocrinol. 2008;158:631-41.

29. Cioffi G, Faggiano P, Lucci D, Maggioni AP, Manicardi V, Travaglini $A$, et al. Left ventricular dysfunction and outcome at two-year follow-up in patients with type 2 diabetes: The DYDA study. Diabetes Res Clin Pract [Internet]. 2013;101:236-42. Disponible en: https://doi.org/10.1016/j.diabres.2013.05.010.

30. Azimova K, San Juan Z, Mukherjee D. Cardiovascular safety profile of currently available diabetic drugs. Ochsner J [Internet]. 2014;14:616-32. Disponible en: http://www.pubmedcentral. nih.gov/articlerender. fcgi?artid=4295739\&tool=pmcentrez \&rendertype=Abstract.

31. Elena del Olmo González, Margarita Carrillo Pérez, Susana Aguilera Gumpert. Actualización del tratamiento farmacológico de la diabetes mellitus tipo 2. Inf Ter Sist Nac Salud. 2008;32:1-16.

32. Prospective UK, Study D. Intensive blood-glucose control with sulphonylureas or insulin compared with conventional treatment and risk of complications in patients with type 2 diabetes (UKPDS 33). 1998;352:837-53 (Ukpds 33).

33. Jones CL. Comparative effectiveness of sulfonylurea and metformin monotherapy on risk of cardiovascular events in type 2 diabetes mellitus. 2015;33:395-401.

34. Cleveland JC, Meldrum DR, Cain BS, Banerjee A, Harken AH. Oral sulfonylurea hypoglycemic agents prevent ischemic preconditioning in human myocardium. Two paradoxes revisited. Circulation. 1997;96:29-32.

35. Hanefeld M, Frier BM, Pistrosch F. Hypoglycemia and cardiovascular risk: Is there a major link? Diabetes Care. 2016;39:S205-9.

36. Ministerio de Salud Colombia. Guiá de Práctica Clińica (GPC) para el diagnóstico, tratamiento y seguimiento de la diabetes mellitus tipo 2 en la población mayor de 18 años. Disponible en: http://gpc.minsalud.gov.co/gpc_sites/Repositorio/Conv_637/ GPC_diabetes/GPC_diabetes_tipo2_completa.aspx.

37. Schramm TK, Gislason GH, Vaag A, Rasmussen JN, Folke F, Hansen ML, et al. Mortality and cardiovascular risk associated with different insulin secretagogues compared with metformin in type 2 diabetes, with or without a previous myocardial infarction: A nationwide study. Eur Heart J. 2011;32:1900-8.

38. Green JB, Bethel MA, Armstrong PW, Buse JB, Engel SS, Garg J, et al. Effect of Sitagliptin on Cardiovascular Outcomes in Type 2 Diabetes. N Engl J Med [Internet]. 2015;373:232-42. Disponible en: http://www.nejm.org/doi/10.1056/NEJMoa1501352.

39. Committee A, Document B, Substance D. SAVOR Saxagliptin Assessment of Vascular Outcomes Recorded in Patients with Diabetes Mellitus sNDAs for Onglyza (22-350 /S-014) and Kombiglyze XR (200-678 /S-013) Briefing Document for Endocrinologic and Metabolic Drugs Advisory Committee Meeting; 2015.

40. Pfeffer MA, Claggett B, Diaz R, Dickstein K, Gerstein HC, Køber LV, et al. Lixisenatide in patients with type 2 diabetes and acute coronary syndrome. N Engl J Med [Internet]. 2015;373:2247-57. Disponible en: http://www.nejm.org/ doi/10.1056/NEJMoa1509225.

41. Marso SP, Daniels GH, Brown-Frandsen K, Kristensen P, Mann JFE, Nauck MA, et al. Liraglutide and cardiovascular outcomes in type 2 diabetes. N Engl J Med [Internet]. 2016;375:311-22. Disponible en:: http://www.nejm.org/ doi/10.1056/NEJMoa1603827.

42. Marso SP, Bain SC, Consoli A, Eliaschewitz FG, Jódar E, Leiter LA, et al. Semaglutide and cardiovascular outcomes in patients with type 2 diabetes. N Engl J Med [Internet]. 2016;375:1834-44. Disponible en:: http://www.nejm.org/ doi/10.1056/NEJMoa1607141.

43. Holman RR, Bethel MA, George J, Sourij H, Doran Z, Keenan J, et al. Rationale and design of the EXenatide Study of Cardiovascular Event Lowering (EXSCEL) trial. Am Heart J [Internet]. 2016;174:103-10. Disponible en:: https://doi.org/10.1016/j.ahj.2015.12.009.

44. Abdul-Ghani M, Del Prato S, Chilton R, De Fronzo RA. SGLT2 inhibitors and cardiovascular risk: Lessons learned from the EMPA-REG Outcome study. Diabetes Care. 2016;39:717-25.

45. Mahaffey KW, Zeeuw De D, Fulcher G, Erondu N, et al. Canagliflozin and cardiovascular and renal events in type 2 diabetes. 2017:1-14. Ph D, Ph D. 